\title{
Derecho y territorio. Una mirada filosófica'.
}

\author{
Law and territory. A philosophical glance
}

Numas Armando Gil Olivera²

RESUMEN

En el presente artículo se abordará desde una perspectiva filosófica la profunda relación que existe entre derecho y territorio, especialmente del hombre como sujeto de derechos con unas cosmovisiones propias dentro de un espacio vital donde desarrolla su ser, en el que encuentra su ethos. Analizando esta interacción inicialmente desde la filosofía del derecho, pasando por la filosofía del ser y a la postre por la filosofía popular - costumbrista caribe.

Palabras clave:

Derecho, territorio, ser y espacio, ethos, costumbre.

\begin{abstract}
In the present article we will approach from a philosophical perspective the profound relationship that exists between law and territory, especially of man as a subject of rights with his own worldviews within a vital space where he develops his being, in which he finds his ethos. Analyzing this interaction initially from the philosophy of law, through the philosophy of being and ultimately by popular philosophy - Caribbean custom.
\end{abstract}

\section{Key words:}

Law, territory, being and space, ethos, custom.

\footnotetext{
${ }^{1}$ Articulo presentando en el marco del seminario sobre debates y perspectivas en torno a la democracia, ciudadanía y los derechos, Facultad de ciencias jurídicas de la Universidad del Atlántico. Año 2016.

${ }^{2}$ Profesor de Filosofía de la Universidad del Atlántico. Presidente de la Asociación Colombiana de Filosofía el Derecho y Filosofía del Social (ASOFIDES) Director del Grupo de Investigación Cronotopias. Correo: mochuelosccantores@yahoo.com / www.nago-filocultura.com

ISSN: 2346-2787

Recibido: 10 de febrero de 2018

Aprobado: 1 de mayo de 2018.
} 
"... El derecho es obra humana, obra de la cultura y en la cultura". (Broekman, 1997)

\section{Introducción}

En Nuestra Cultura jurídica la propia raíz etimológica de los términos: "lus, Right, Recht, Droit, Deritto, derecho". Evoca y se remite a exigencias de rectitud, corrección, racionalidad, proporción; en suma de justicia.

El profesor Norberto Bobbio, argumenta que el significado que está más, estrechamente conectado con la teoría del estado o de la política es el del derecho, como ordenamiento normativo. Es decir, como conjunto de normas de conducta y organización que constituyen una realidad, que tienen por contenido la reglamentación de relaciones fundamentales para la convivencia y la supervivencia del grupo social, como son las relaciones familiares, las relaciones económicas, las relaciones superiores de poder; así como la reglamentación de los modos y de las formas de primer grado o institucionalización de la sanción, y que tiene como fin mínimo el impedimento de la acciones consideradas más destructiva del conjunto social, la solución de los conflictos que amenazan, si no son resueltos, con hacer imposible la propia subsistencia del grupo, la consecución y el mantenimiento.

En suma, del orden o de la paz social... el derecho recurre en última instancia a la fuerza física para obtener el respeto de las normas, para ser eficaz el ordenamiento en su conjunto...la conexión entre derecho y política se hace tan estrecha como para hacer considerar al derecho como el principal instrumento mediante el cual la fuerzas políticas que detienen el poder dominante en una determinada sociedad ejercen su dominio. (Bobbio, 1991) 
De esta conexión se hizo consiente la filosofía política y jurídica que acompaña el nacimiento del estado moderno e interpreta y refleja su espíritu, desde Hobbes, a través de Locke, Rousseau, Kant, Hegel, Marx, hasta Max Weber, Kelsen y Kaufmann; al punto de hacer aparecer estructura jurídica y poder político, ordenamiento y fuerza coactiva, el momento de la organización de las fuerzas para alcanzar sus propios objetivos.

Para un abogado "continental", - de la tradición de Europa Continental, es decir, de la tradición romano- germánica, contraria a la tradición británica del common Law, el derecho, es un sistema de normas autónomas, racional, consistente y coherente. La principal fuente del Derecho es la legislación expedida mediante un procedimiento jurídicamente regulado. Los insumos legislativos son interpretados y sistematizados por académicos del derecho y aplicados a casos concretos por los jueces.

El derecho también es un sistema de procedimientos mediante el cual las normas son establecidas, aplicadas, interpretadas y sistematizadas. Podemos considerar estos procedimientos como discursos en un sentido muy específico del término. Los discursos son procedimientos en los que las relaciones de poder están excluidas por definición. El criterio del mejor argumento es el único poder operativo en los discursos. A cualquiera se le permite participar en igualdad de condiciones para expresar sus necesidades, intereses y argumentos.

En el nivel jurisdiccional, tenemos discursos de aplicación, donde las normas justificables son aplicadas sobre la base de una descripción del caso concreto, que cubre los aspectos relevantes de dicho caso. Finalmente, tenemos discursos entre expertos del derecho, en los que se resuelven las eventuales inconsistencias internas o incoherencias entre las normas válidas. El autor de esa narrativa, como dirían los posmodernos- es Jürgen Habermas, Robert Alexy, Klaus Günther y Manuel Atienza. 
El derecho también lo argumentan como un instrumento al servicio de aquellos que detectan el poder político. Quienes tienen ese poder pueden ser caracterizados tanto como los miembros de una clase dominante o como los vencedores de una competencia limpia por posiciones gubernamentales. Han existido dos visiones de la historia: la revolucionaria y la reformista, que sin embargo comparten los mismos sentidos instrumentalistas del derecho.

Podríamos argumentar, que el derecho obedece a la racionalidad de la norma y produce efectos características de las tecnologías de poder, utilizando la fuerza de la norma. Este punto de vista, fue desarrollado en primer lugar por Michel Foucault y retomado por Francois Ewald en su Genealogía del Estado de bienestar y del derecho social. (Ewald, 1986)

Lo que el enfoque del Derecho como argumentación trata de hacer es conectar todos los elementos de análisis a partir de una concepción dinámica, instrumental y "comprometida" del derecho que arranca de la noción de conflicto.

El conflicto es, en efecto, el origen del derecho, lo que lleva a verlo como un instrumento, una técnica de tratamiento de problemas de cierto tipo. Además, una característica del Derecho contemporáneo, es que las tomas de decisión en relación con los conflictos tienen que estar sustentadas por razones de cierto tipo, por argumentos. Por tanto, el derecho puede verse como una compleja institución volcada hacia la resolución de conflictos por medios argumentativos y en las diversas instancias de la vida jurídica (Atienza, 2004).

En el concepto de derecho: H.L.A. Hart dice que el derecho exhibe una variedad o diversidad de normas no siempre reductibles al esquema simple de las prescripciones o normas de conducta, que ordenan, prohíben o permiten determinados comportamientos. (Hart, 1961 traducido por Carrió, 1998) 
Hart se adscribe a una concepción del derecho que ve o considera a este en términos de una realidad normativa. En síntesis; - el concepto de derecho- se ofrece según propias palabras de Hart, "como una elucidación del concepto de derecho y no como una definición de derecho".

Todas estas argumentaciones como una proximidad al concepto de derecho; es con el único fin de que sin él, no se puede concebir el territorio. O mejor, Territorio y Derecho; está habitado por un sujeto, que arrastra su condición humana en un territorio.

\section{Ethos Y Territorio.}

Recordemos que nuestra Carta Política de 1991, desde su primer articulado está a favor del Estado Social de Derecho. Está "organizado en forma de república unitaria, descentralizado, con autonomía de sus entidades territoriales, democrático, participativo y pluralista, fundado en el respeto de la dignidad humana, en el trabajo y la solidaridad de las personas que lo integran y en la prevalencia del interés general".

Es nuestra Carta política, el Territorio es autónomo. Y en las últimas décadas, se ha convertido en una de los más citados en escenarios muy diferentes y por actores muy variados, desde académicos hasta organizaciones de base, pasando por formulaciones de políticas públicas y por directivos de empresas transnacionales.

"Esta relativa nueva preocupación con el territorio ha encontrado en el tema del ordenamiento territorial uno de sus escenarios predilectos". Es así que se han venido planteando perspectiva que proponen comprender la dimensión territorial como articuladora de las demás dimensiones sociales. Como por ejemplo: 
- En lo político, se experimenta una tendencia a la generalización de la democracia representativa occidental como la forma de gobierno. "ideal" y al mismo tiempo el surgimiento de nuevos actores de escalas diferentes a la nacional.

- En lo económico, se ha intensificado el nivel de los flujos e intercambios comerciales a escala mundial, de manera que hay una orientación de los modelos económicos nacionalistas hacia las exportaciones y la inversión extranjera directa.

- En lo cultural, se identifica la intención de generalizar la cultura Pop occidental como ideal de comportamiento individual, ligado con la emergencia de interpretaciones pos-modernas de la sociedad.

La distancia física se reduce para acelerar el periodo de retorno mercantil. Lo que ha llevado a generaciones sucesivas de resoluciones en el ámbito técnicocientífico- informacional, llegando en la actualidad a la posibilidad de una conexión instantánea entre lugares separados de miles de kilómetros. De esta manera se ha creado un discurso según el cual, el espacio y el tiempo han sido contraídos gracias a la velocidad, representando "la muerte del espacio" por el capital.

Sin embargo, en este nuevo engendro global "el espacio geográfico gana nuevos contornos, nuevas características, nuevas definiciones. $Y$ también nueva importancia, porque la eficacia de las acciones está estrechamente relacionada con su localización. Entonces, inferimos que la insurgencia de lo territorial se interpreta como una consecuencia de la crisis o vaciamiento del "Estado Nación". (Olmedo-Martínez, 2010).

En esta nueva dinámica no existe una única dirección de las acciones sociales, es decir, las condiciones globales no imponen su lógica a las prácticas locales, ni estas últimas son autónomas del nivel global; lo que ocurre es una profundización de la interdependencia entre movimientos de diferentes escalas. Como los Uwa, Los Nasa del Cauca, Los pueblos de La Sierra Nevada, etc.

"Sin espacio no hay ser", Plantea Henri Lefebvre. Esta reflexión significa que todo sucede en un espacio. Todas las acciones, fenómenos y procesos se despliegan 
en espacio, la dimensión espacial es parte constituyente de la sociedad y que cada sociedad produce, organiza y se relaciona física y simbólicamente con el espacio de acuerdo con particularidades históricas y culturales (Lefebvre, 1991)

De ahí que el Ethos es como el destino del hombre. Es decir, se argumenta que en su origen más arcaico ethos significó "morada" o "guardia" de los animales, y que solo más tarde, por extensión, se refería al ámbito humano, conservando de algún modo, ese primigenio sentido de "lugar y resguardo", de refugio o protección; de "espacio" vital seguro, cubierto de la "intemperie" y en el cual se acostumbra "habitar".

El sentido de "habitar" o "morar" está ciertamente entrañado en el ethos humano: remite a la idea esencial de "morada interior". El ethos es "lugar" humano de "seguridad" existencial (autarquía). Aunque también lo significativo es que se trata de un lugar acostumbrado, habitual, familiar. De ahí que ethos signifique también costumbre, uso. Remita a una forma habitual de comportamiento. Y de ahí también su asociación al término hábito o costumbre. $Y$ en tanto que "hábito: acción continuada o reiteración de una conducta ("habituar"), el ethos remite, no ya a un lugar o espacio sino más bien al tiempo, a la continuidad temporal. Aunque quizá sea más preciso decir que en el ethos, particular, se expresa de manera eminente la condición espacio temporal del hombre.

De esa idea de "consistencia" existencial derivaría la noción esencial del ethos como "carácter", en el sentido literal de modo de ser, disposición ("carácter moral", "modo ético de ser"). En tanto que disposición o actitud, es forma de estar ante el mundo, ante otros: forma de relación (de "recibir" y "dar"). El ethos hace patente la condición "relativa" del hombre (su ser-en-relación). Así mismo el ethos revela que el ser para el hombre es, en efecto, "modo o "manera" o "forma de ser", cualitativamente diferenciada; el "cómo" de vivir humano resulta determinante del propio ser. $\mathrm{Y}$ el ethos revela también que la "manera de ser" depende de una acción (hábito) y, por tanto, no es algo dado, sino creado, generado por la propia 
acción. El ethos sin duda lleva la idea de estabilidad, consistencia, persistencia, fidelidad a si mismo e "identidad" temporal.

Cuando Heráclito afirma: "el ethos es para el hombre su daimon", está afirmando ciertamente que "el carácter" es para el hombre su destino, entendiendo destino, como meta o finalidad última (telos). Los fines éticos son ciertamente, meta propia de la vida humana (González, 1996).

Resumiendo podríamos decir que el ethos, es el carácter, la residencia, la morada; el lugar donde habitan los animales. El suelo firme donde brotan los actos humanos. Es el modo de ser, su costumbre en la vida que se va apropiando mediante la existencia, una determinada manera de comportarse el ser en el territorio. El ethos se expresa en el deporte, vestir, andar, comer, o a través de la calma de cualquier suceso en el Territorio.

\section{La costumbre se convierte en ley}

Al comienzo del libro II, de la Ética nicomaquea, Aristóteles va a distinguir dos clases de virtud, la "día noética" y la "ética". La primera "debe su origen y su incremento principalmente a la enseñanza, y por eso requiere experiencia y tiempo". La segunda, "en cambio, procede de la costumbre (ethos)". (Aristóteles, Ética nicomaquea, 1953).

En el caribe la transparencia epistemológica no ha desplazado las borras y pasos en los arcanos cosmogónicos, las aspersiones de sangre propias del sacrificio, sino que, a diferencia de los que ocurre en occidente, el conocimiento científico y el conocimiento tradicional coexisten en estado de diferencias.

La máquina caribeña es una máquina de flujo y de interrupción a la vez; es una maquina tecnológico- poética. O si se quiere, una meta máquina de diferencias, 
cuyo mecanismo poético no puede ser diagramado en las dimensiones convencionales, y cuyas instrucciones se encuentran dispersas en estado de plasma dentro del caso de su propia red de códigos y sub-códigos.

El ritmo, en los códigos del caribe, precede a la música, incluso de la misma percusión. Es algo que ya estaba ahí, en medio del ruido; algo antiquísimo y oscuro a lo cual se conecta en un momento dado la mano del tamborero y el cuero del tambor; una suerte de chivo expiatorio, ofrecido en sacrificio, que se puede entrever en el aire cuando uno se deja llevar por el sonido milenario de un conjunto de gaitas o chuanas. O el trucuteo que se siente en el alma al escuchar porros y tamboreos batá a cuyo ritmo bailan los vivos y los muertos. Se trata de un meta ritmo al cual se puede llegar por vía, de cualquier sistema de signos, llámese este música, lenguaje, arte, texto, danza, etc. Digamos, uno empieza a caminar y de repente se da cuenta que está caminando "bien", es decir, no solo con los pies, sino con otras partes del cuerpo; cada musculo se mueve sin esfuerzo, a un ritmo dado, y sin embargo se ajusta admirablemente al ritmo de los pasos.

\section{Conclusiones.}

En el caribe lo "extranjero" interactúa con lo "tradicional" como un rayo de luz con un prisma; esto es, se producen fenómenos de reflexión, refracción y descomposición, pero la luz sigue siendo luz: "la memoria es siempre una forma del presente, pero no por consolación o equivalencia sino porque el tiempo es la materia que construye al Caribe; y en ese canje permanente, la memoria es un telón de fondo y el presente su orilla decorada. El tiempo se demora en las calles, rostros, lecturas, conversaciones, testigos y testimonios, de una vida consiente de transito de abundancia y nostalgia de ironía y tolerancia". 
El caribe es ese sitio, donde el no poder salir, es solo la forma más extrema de no haber llegado nunca. Lo característico del ser caribeño es que, en lo fundamental, su experiencia estética ocurre en el marco de rituales y representaciones de carácter colectivo, histórico e improvisatorio.

Mientras no tomemos conciencia de ser poseedores intelectuales de nuestro pasado arqueológico y artístico, mientras no podamos trabajar creativamente en las ciencias, mientras no formemos expertos familiarizados con las técnicas y basados en ellas, mientras no podamos explotar autónomamente nuestros recursos naturales, mientras sigamos a merced de la sensibilidad y la emoción y no poseamos ideas claras sobre nuestro ser, sobre nuestro mundo físico e histórico, seguiremos dependiendo del exterior, seguiremos siendo una fuente de materia prima, un mercado y un objeto de explotación.

Mientras el hombre del caribe no tome posesión intelectual de su pasado nunca se realizara esa "amenaza" de Héctor Rojas Herazo, de ver el nacimiento de una metafísica para el tercer mundo. Eso que él llama la posibilidad de lo americano como expresión, la salvación posible por el asombro, único instrumento mágico para la capacitación superior que nos espera en cada ser, en cada piedra, en cada temblor de la atmosfera americana... lo que vive palpitando, lo que nos invade, los ayeres sin hoy y los mañanas apretados en un tambor, mugre y esperanza. ¡Sapere aude!

\section{Referencias.}

Aristóteles (1953) obra clásica: Ética nicomaquea, libro II (1103 A 15), Biblioteca Clásica de Gredos: Madrid. 
Atienza, M. (2004) El derecho como argumentación. Distribuciones Fontanara: México.

Bobbio, N. Diccionario de Política. Siglo XXI; 13a․ Edición México.

Broekman. J. M. (1997) Derecho, filosofía del derecho y Teoría del Derecho. Temis: Bogotá.

Ewald, F. (1986). L’Etat providence. París: Grasset.

González, J. (1996) El ethos, destino del hombre. F.C.E. México.

Hart, Herbert L. A. (1961) The Concept of Law. Oxford, Clarendon Press. traducción castellana de Genario R. Carrió (1998) El concepto de derecho, Ed. Abeledo-Perrot: . Buenos Aires.

Lefbvre, H. (1991) Še production of space. Oxford: Blackwell.

Olmedo-Martinez, L. (2010) Visiones con-partidas del territorio. En un mundo dividido. Universidad Nacional: Bogotá. 